\title{
Genetic influences on fibrinogen, tissue plasminogen activator- antigen and von Willebrand factor in males and females
}

\author{
Marlies de Lange', Eco J. C. de Geus', Cornelis Kluft ${ }^{2}$, Piet Meijer², Lorenz J. P. van Doornen³, Dorret I. Boomsma', \\ Harold Snieder ${ }^{4,5}$ \\ 'Department of Biological Psychology, Vrije Universiteit, Amsterdam, The Netherlands \\ ${ }^{2}$ Gaubius Laboratory, TNO Quality of Life, Leiden, The Netherlands \\ ${ }^{3}$ Department of Health Psychology Universiteit Utrecht, Utrecht, The Netherlands \\ ${ }^{4}$ Georgia Prevention Institute, Department of Pediatrics, Medical College of Georgia, Augusta, Georgia, USA \\ ${ }^{5}$ Twin Research and Genetic Epidemiology Unit, St Thomas' Hospital, London, United Kingdom
}

\begin{abstract}
Summary
Differences in genetic influence on death from CHD between males and females have been reported. Haemostatic factors have consistently been associated with risk for coronary heart disease (CHD), but sex differences in genetic architecture have not been studied. This study in middle-aged twins investigates whether there are sex differences in means and in genetic and/ or environmental variance components of haemostatic risk factors for CHD. A total of 93 monozygotic twin pairs (44 male and 49 female) and I I 6 dizygotic twin pairs ( 36 male, 40 female and 40 opposite sex) were available for this study. Structural equation modelling was used to estimate the relative influence of genetic and environmental factors on variation in levels of fi-
\end{abstract}

\section{Keywords}

Twins, haemostasis, heritability, sex differences, oral contraceptive pill

\section{Introduction}

The genetic influence on death from CHD in males and females has been investigated in a large Swedish twin sample and a heritability of $38 \%$ was estimated for females, which was notably lower than the $57 \%$ estimated for males (1). The Swedish twin sample included only same-sex twin pairs precluding any inference on qualitative differences in genetic influences on death from CHD, i.e. whether genes contributing to CHD in women are distinct from genes contributing to CHD in men. Sex differences in the genetic architecture of CHD risk factors may hold the key in explaining the difference in heritability of CHD mortality and deserve closer study than has been performed so far. brinogen, tissue plasminogen activator (tPA) antigen and von Willebrand factor (VWF). Mean levels of tPA and VWF increased with age. Oral contraceptive pill (OCP) and menopause had significant influences on levels of fibrinogen and tPA. Genetic influences explained 39,66 and $72 \%$ of the variation in levels of fibrinogen, tPA and vWF, respectively. No quantitative or qualitative differences of genetic influences on haemostatic levels were seen between males and females. Haemostatic factors may account for a significant part of the genetic risk for cardiovascular disease. No difference in genetic architecture for levels of fibrinogen, tPA or vWF was observed between males and females.
Only a small proportion of the genetic influence on death from CHD could be accounted for by traditional risk factors such as smoking, hypertension, obesity and diabetes $(2,3)$, indicating the existence of other (genetic) risk factors for CHD. Here we hypothesize that a genetically driven disturbance in the balance of coagulation and fibrinolysis will explain part of the genetic variance in CHD. Such an imbalance in haemostasis constitutes an established risk factor for the development of atherosclerosis and subsequent cardiovascular disease $(4,5)$. Large prospective studies have shown elevated haemostatic factors such as fibrinogen, tissue plasminogen activator (tPA) antigen, plasminogen activator inhibitor type 1 (PAI-1) and von Willebrand factor $(\mathrm{vWF})$ as risk factors for cardiovascular disease (6-12). Interest- 
ingly, it was previously found that fibrinogen levels were higher in females compared to males and only become more similar between the ages of 55 and 64 (13). Similarly, higher levels of vWF have been observed in females compared to males in both patients with acute myocardial infarction (14) and in individuals free from symptoms of cardiovascular disease (15). In contrast, levels of tPA antigen have been found to be lower in females compared to males (16). It is unclear to what extent these differences in mean levels of haemostatic risk factors relate to sex differences in ultimate risk for cardiovascular disease.

Understanding whether sex differences in risk factors are due to genetic differences between males and females would help molecular biological studies unravel the pathways of the (differential) development of cardiovascular disease. Haemostatic risk factors have shown moderate to large genetic influences, but none of these studies investigated sex differences in genetic influence on haemostatic risk factors (17-19). The study of male and female as well as opposite-sex twin pairs provides a unique opportunity to investigate sex differences in genetic and/or environmental influences on variation of haemostatic risk factors. A higher resemblance in monozygotic (MZ) compared to dizygotic (DZ) twin pairs, who share $100 \%$ and (on average) $50 \%$ of their DNA, respectively, provides the first indication of the genetic component of a trait. Using same-sex twin pairs, sex differences in magnitude of genetic and environmental effects can be tested by comparing this excess similarity in MZ twins between males and females. DZ opposite sex pairs additionally allow testing for qualitative sex differences, i.e. whether genes contributing to haemostatic factors in women are different from those in men.

This study investigates in a sample of 213 middle-aged male and female twin pairs whether there are sex differences in the genetic architecture of fibrinogen, tPA antigen and vWF.

\section{Materials and methods}

\section{Subjects}

Subjects were part of a study at the Netherlands Twin Registry on familial clustering of cardiovascular risk factors in middle-aged twins (20). A total of 426 twins [96 MZ and $117 \mathrm{DZ}$ pairs] aged 34 to 62 had a blood sample taken after an overnight fast. This baseline sample was taken at the start of a two and a half hour experimental protocol consisting of mental stressors interspersed with quiet resting and filling out a series of questionnaires on demographics, lifestyle and mental health (20). This study was approved by the local ethics committee, and written informed consent in accordance with the Helsinki declaration was obtained from all participants.

Exclusion of twins with known (cardio)vascular disease $(n=9)$ had virtually no effect on twin correlations. Therefore, participants were only excluded from the analysis if they were on oral anticoagulant therapy $(\mathrm{n}=1)$. Of $3 \mathrm{MZ}$ twin pairs, the samples of one twin were lost at processing, and the data of one DZ twin was excluded from the analysis due to oral anticoagulant therapy, resulting in 93 complete MZ twin pairs and $116 \mathrm{DZ}$ twin pairs for the analysis of tPA antigen and vWF. Of one additional MZ twin, the sample of fibrinogen was lost resulting in 92 complete pairs available for analysis of fibrinogen. Incomplete twin pairs were left in the analysis for their contribution to the estimation of variance. In part of the sample, a second blood sample was taken after the completion of the entire experimental protocol as previously described (21). A second measure of fibrinogen, IPA antigen and vWF from a blood draw at the end of the experimental protocol was available for 105 twin pairs [60 complete $\mathrm{MZ}$ and 45 complete $\mathrm{DZ}$ twin pairs] and 3 single members of an MZ twin pair and 1 member of a DZ twin pair. These data were also used in the estimation of the variance components (see below).

\section{Blood sampling and biochemical assays}

Venous blood samples were taken by venipuncture using Vacutainer tubes containing sodium-EDTA (Becton-Dickinson). The first sample was taken around $10.45 \mathrm{am}$, the second one around $1.30 \mathrm{pm}$. Tubes were placed on ice and centrifuged within $30 \mathrm{~min}$ at $4^{\circ} \mathrm{C}$ to separate plasma from cells. Aliquots of plasma were snap-frozen using liquid nitrogen and stored at $-20^{\circ} \mathrm{C}$ until further analysis. The average timespan from blood sampling to analysis was 3 months. Fibrinogen was determined by enzymelinked immunosorbent assay (ELISA; Organon Teknika, Turnhout, Belgium) as described by Hoegee-de Nobel, et al. (22) Determination of levels of tPA antigen was carried out by ELISA (Organon Teknika, Turnhout, Belgium) as described by Bos, et al. (23). vWF was assessed by ELISA, using polyclonal antibodies (Dako; Cat. No. A082).

\section{Statistical analysis}

All variables were logtransformed prior to analysis to obtain normal distributions, avoiding bias in the maximum likelihood model fitting results. Preliminary results were obtained using the statistical software package STATA (24). The resemblance in twins is expressed by Pearson's correlations. A batch effect was detected for fibrinogen, where the mean of the first 7 batches was lower than the mean of the following 20 batches. To adjust for this batch effect prior to analysis, the difference in means between the first 7 and following 20 batches was added to the fibrinogen levels of the first 7 batches. The effect of oral contraceptive pill (OCP), menopause, and sex on levels of fibrinogen, tPA and von Willebrand was tested using generalised estimation equation (GEE) which allows to take into account the non-independence of the twin data. Eight and a half percent $(\mathrm{N}=19)$ of the female twins had a hysterectomy. This group was coded to a separate category in the menopause variable (i.e., premenopausal, hysterectomy, postmenopausal). The effect of age, OCP, menopause and sex were subsequently controlled for in genetic analysis (see below).

\section{Genetic model fitting}

Twin studies are an ideal method to quantify genetic and environmental influences on complex traits such as risk factors for cardiovascular disease. MZ twins share $100 \%$ of their genes, whereas DZ twins share on average $50 \%$ of their genes, like ordinary siblings. Under the assumption that both types of twins share their common (family) environment to the same extent, a greater similarity in MZ twins compared with DZ twins reflects genetic influences. Comparing this excess similarity in MZ twins between male-male and female-female twins and contrasting simi- 


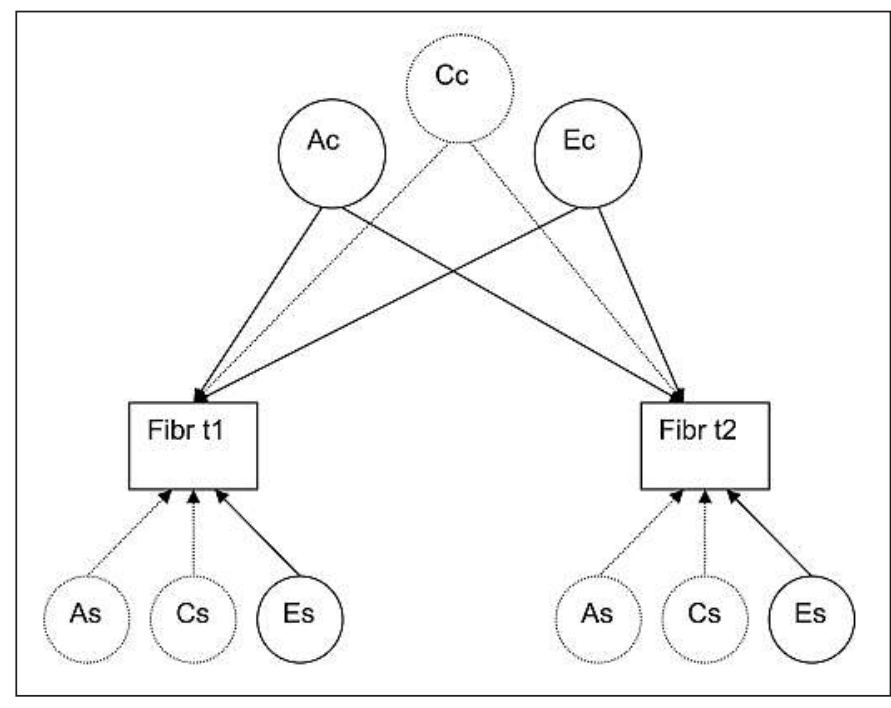

Figure I: Independent pathway model for fibrinogen at two time points $(\mathbf{t l}, \mathbf{t 2})$. The boxes represent the measured phenotypes, and the circles the latent factors. Ac is the additive genetic influence common for both time points, $\mathrm{Cc}$ is the shared environmental influence and $\mathrm{Ec}$ is the unique environmental influence common to both time points. As, Cs and Es are the additive genetic, shared environmental and unique environmental effects, respectively, specific to each of the time points. For clarity the information of only one twin is shown. The shaded latent factors and pathways were not significant for either fibrinogen, tPA nor vWF. To avoid under-identification of the model, the paths from Ac to timepoints I and 2 are estimated to be equal, as are the paths from Ec to timepoints I and 2.

larity of same-sex DZ twins to opposite sex (OS) twins allows testing of possible differences in genetic influences between females and males.

Model-fitting techniques of twin data make optimal use of the information available in the twin co-twin covariance structure. These techniques are based on the comparison of observed and expected variance-covariance matrices (25). The phenotypic variances are assumed to be linear functions of the underlying additive genetic variance (A), common (or shared) environmental variance $(C)$, and unique environmental variance $(E)$, which also contains measurement error, so that the total (or phenotypic) variance (Vp) equals: $\mathrm{A}+\mathrm{C}+\mathrm{E}$. Division of each of these components by the total variance gives the different standardised components of variance, for example the heritability $\left(\mathrm{h}^{2}\right)$ which can

Table I: Means (SD) of age and untransformed coagulation factors for males vs. females.

\begin{tabular}{l|c|c|c|c} 
& \multicolumn{2}{|c|}{ Males } & \multicolumn{2}{c}{ Females } \\
\hline $\mathrm{N}$ & \multicolumn{2}{|c|}{203} & \multicolumn{2}{c}{219} \\
\hline Age (yrs) & 43.7 & $(6.48)$ & 44.7 & $(6.76)$ \\
\hline Smokers (\%) & \multicolumn{2}{|c|}{34} & \multicolumn{2}{|c}{31} \\
\hline Fibrinogen (g/l) & 2.57 & $(0.49)$ & 2.71 & $(0.54)^{*}$ \\
\hline tPA antigen (ng/ml) & 8.68 & $(3.40)$ & 6.99 & $(4.14)^{*}$ \\
\hline VWF (\% pooled plasma) & 129.4 & $(45.96)$ & 132.7 & $(49.98)$ \\
\hline
\end{tabular}

$\mathrm{N}=$ number of individuals; * Using GEE, the male - female difference $\mathrm{p}$-value $<0.05$. smoker $=$ smoking one or more cigarettes a day. be defined as the proportion of overall phenotypic variation that can be explained by additive genetic factors. The significance of variance components $\mathrm{A}$ and $\mathrm{C}$ was assessed by testing the deterioration in a model fit after each component was dropped from the full model (ACE), leading to a parsimonious model in which the pattern of variances and covariances is explained by as few parameters as possible. Standard hierarchic $\chi^{2}$ tests were used to select the best-fitting model.

Differences in heritability between sexes can arise in two ways. First, quantitative differences would arise when there is a difference in the magnitude of genetic (and/or environmental) effects on the coagulation factors between the sexes. In this case, the full model where the genetic effect for males and females is estimated separately is compared with a model where the genetic effect for males and females is estimated to be the same. For example, when genetic influences have a greater influence on males than females, the submodel where the genetic effects are constrained between males and females would show a deterioration in fit compared to the full model where these effects are estimated separately for the sexes. Second, qualitative differences would arise when there are different genes at work in males compared to females. When this would be the case, a significantly lower OS twin correlation compared to the expected 0.5 correlation for $\mathrm{DZ}$ would be observed. This smaller genetic relationship among OS twins compared to their same-sex DZ counterparts indicates that different genes are expressed in males and females. Genetic differences were tested in a univariate genetic analysis of the baseline coagulation measures, i.e., before the mental stress tasks.

As mentioned above, two measures of the coagulation factor, before and after a two and a half hour experimental protocol including several mental stress tasks, were available in part of the twin sample. To make optimal use of the data, but to allow for any effect of the stress task on the coagulation levels, these were modelled in bivariate (independent pathway) analyses. The effect of age, OCP, menopause and sex on mean levels of the coagulation factors were controlled for within the variance-covariance modelling framework. Separate means were estimated for males and females. For females, OCP and menopause were allowed to have an effect on the mean values. Estimation of the regression coefficient for age was done within sex. The interaction of age and sex was tested by comparing whether the age coefficients of males and females could be constrained to be equal after controlling for the effect of OCP and menopause in females. Subsequently this model was simplified to obtain the most parsimonious solution (see Fig. 1).

Models were fitted to the raw data using normal theory maximum likelihood allowing the use of information provided by unpaired (i.e., single) twin observations. Estimates of the genetic and environmental variance components and the appropriate $95 \%$ confidence interval $(\mathrm{CI})$ were obtained from the best-fitting model. All model fitting was carried out in the structural equation modelling software Mx (26).

\section{Results}

Table 1 presents the general characteristics of the twins. There was no difference in mean age, nor in percentage of smokers be- 
Table 2: Means (SD) of age and untransformed coagulation factors for $M Z$ vs $D Z$ twins.

\begin{tabular}{|l|c|c|c|c|}
\hline & \multicolumn{3}{|c|}{ MZ } & \multicolumn{2}{c|}{ DZ } \\
\hline $\mathrm{N}$ & \multicolumn{2}{|c|}{93} & 44.2 & $(6.66)$ \\
\hline Age $(\mathrm{yrs})$ & 44.2 & $(6.63)$ & 2.65 & $(0.54)$ \\
\hline Fibrinogen $(\mathrm{mg} / \mathrm{ml})$ & 2.64 & $(0.5 \mathrm{I})$ & 8.17 & $(4.50)$ \\
\hline tPA antigen $(\mathrm{ng} / \mathrm{ml})$ & 7.35 & $(2.91)$ & 129.3 & $(51.18)$ \\
\hline VWF $(\%$ pooled plasma) & 133.3 & $(43.93)$ & & \\
\hline $\mathrm{N}=$ number of complete twin pairs. & & & \\
\hline
\end{tabular}

Table 3: Means (SD) of age and untransformed coagulation factors by sex, OCP, hysterectomy and menopausal status.

\begin{tabular}{|l|c|c|c|c|c|c|c|c|c|}
\hline & N & \multicolumn{2}{|c|}{ Age } & \multicolumn{2}{c|}{ Fibrinogen } & \multicolumn{2}{|c|}{ tPA } & \multicolumn{2}{c|}{ vWF } \\
\hline Males & 203 & 43.7 & $(6.48)$ & 2.57 & $(0.49)$ & 8.68 & $(3.40)$ & 129.4 & $(45.96)$ \\
\hline F- OCP & 27 & 39.8 & $(5.1 \mathrm{I})$ & $2.8 \mathrm{I}$ & $(0.45)$ & 4.35 & $(1.88)$ & 140.2 & $(46.21)$ \\
\hline F-no OCP & 125 & 41.9 & $(4.02)$ & 2.59 & $(0.54)$ & 6.40 & $(2.40)$ & 125.2 & $(46.38)$ \\
\hline F- hyst & 19 & 47.0 & $(5.67)$ & 2.76 & $(0.53)$ & 6.35 & $(2.74)$ & 126.1 & $(37.36)$ \\
\hline F- postmeno & 48 & 53.6 & $(4.89)$ & 2.96 & $(0.50)$ & 10.26 & $(6.59)$ & 150.6 & $(60.57)$ \\
\hline F- females, OCP oral contraceptive pill.
\end{tabular}

tween either males and females or between $\mathrm{MZ}$ and $\mathrm{DZ}$ twin pairs (see Table 1 and 2). Mean levels of fibrinogen were lower in males compared to females, but mean levels of tPA antigen were higher in males compared to females. No difference in levels of vWF between males and females was observed. As expected, there was no significant difference in mean levels between MZ and DZ twins for any of the coagulation factors (see Table 2).

The mean levels of fibrinogen, tPA and vWF for males, females taking OCP, females not taking OCP, females who had undergone hysterectomy and postmenopausal females are shown in Table 3. For fibrinogen, no clear pattern is seen, apart from slightly higher levels in females taking OCP and in postmenopausal females. For tPA, 32\% lower levels are seen for females taking OCP compared to females not taking OCP, whereas postmenopausal females have $60 \%$ increased levels compared to premenopausal females not taking OCP. Males have $36 \%$ higher levels of tPA compared to premenopausal females not taking OCP. GEE showed that, when taking into account the significant positive effects of OCP $(p=0.03)$ and menopause $(p=0.01)$ on fibrinogen levels, the effect of age is no longer significant, nor is there any difference between males and females. Only the effect of age $(p=0.01)$ was significant for levels of vWF. In the case of tPA, the effect of age, OCP, menopause and male gender were all significant contributors to variation in levels (all p-values $<$ 0.003 ), but no interaction of menopause with age or OCP with age or sex with age could be detected.

Table 4 presents the twin correlations for the zygosity by sex groups. In general, the MZ twin correlations are higher than the DZ twin correlations, suggesting a genetic influence on the coagulation factors. However, for fibrinogen the MZ male correlation of 0.18 seems very low compared to the MZ female correlation (0.43). Fibrinogen is an inflammatory marker and therefore subject to environmental influence. This can make the correlation somewhat instable in small samples. Note, however, that male and female $\mathrm{MZ}$ correlations were not significantly different and jointly resulted in a total $\mathrm{MZ}$ correlation of 0.35 . This joint correlation was significantly higher than the overall DZ correlation of 0.15 .

A small difference in correlation between OS and DZ twins is seen for fibrinogen and vWF. However, the genetic correlation between OS twins was not significantly different from 0.5 (data not shown), indicating that genes for the coagulation factors are not different between males and females. No differences in the magnitude of genetic and environmental influences were found between males and females for any of the coagulation measures in univariate analysis.

Table 4: Means (SD) of untransformed coagulation factors and Pearson's correlation for each zygosity group.

\begin{tabular}{|c|c|c|c|c|c|c|c|c|c|c|}
\hline Zyg & $\mathbf{N}$ & \multicolumn{3}{|c|}{ Fibrinogen } & \multicolumn{3}{|c|}{ tPA } & \multicolumn{3}{|c|}{ vWF } \\
\hline MZM & 44 & 2.54 & $(0.43)$ & 0.18 & 8.12 & $(3.03)$ & 0.71 & 137.6 & $(44.83)$ & 0.74 \\
\hline MZF & 49 & 2.74 & $(0.55)$ & 0.43 & 6.65 & $(2.62)$ & 0.57 & 129.4 & $(42.96)$ & 0.69 \\
\hline$\overline{D Z F}$ & 40 & 2.71 & $(0.56)$ & 0.15 & 7.59 & $(5.74)$ & 0.30 & 135.2 & $(51.26)$ & 0.29 \\
\hline DOS & 40 & 2.61 & $\begin{array}{l}(0.49) \\
\end{array}$ & 0.12 & 7.56 & $(3.24)$ & 0.32 & 131.4 & $(57.15)$ & 0.26 \\
\hline
\end{tabular}

Table 5: Model fitting results of best fitting independent pathway model and their $\mathbf{9 5 \%}$ confidence intervals (95\% $\mathbf{C l})$. See Figure I for explanation of best fitting model. Parameter estimates are shown for the pre-stress measure only.

\begin{tabular}{|c|c|c|c|c|c|c|c|c|}
\hline & Best fitting model & $a^{2}$ & $(95 \% \mathrm{Cl})$ & $e^{2}$ & $(95 \% \mathrm{Cl})$ & p-value $\beta_{\text {age }}$ & p-value $\beta_{\text {ocp }}$ & $\mathrm{p}$-value $\beta_{\text {meno }}$ \\
\hline Fibr & $\mathrm{AE}, \mathrm{Es}_{1}, \mathrm{Es}_{2}$ & 0.39 & $(0.22-0.54)$ & 0.61 & $(0.46-0.78)$ & 0.22 & 0.03 & 0.001 \\
\hline $\mathrm{tPA}$ & $\mathrm{AE}, \mathrm{Es}_{2}$ & 0.67 & $(0.54-0.76)$ & 0.33 & $(0.23-0.46)$ & 0.001 & $<0.001$ & 0.054 \\
\hline vWF & $\mathrm{AE}, \mathrm{Es}_{2}$ & 0.72 & $(0.61-0.80)$ & 0.28 & $(0.20-0.39)$ & 0.003 & 0.075 & 0.18 \\
\hline
\end{tabular}


Table 5 shows the estimates of the variance components and their $95 \%$ confidence intervals from the best fitting independent pathway models (Fig. 1), as well as the significance of beta coefficients of age, OCP and menopause from the structural equation modelling. After taking into account the effect of OCP and menopause, an effect of age could no longer be detected for fibrinogen. For tPA, an effect of age was still present after taking into account OCP and menopause, but could be set equal for males and females, indicating no interaction of sex with age. For vWF only an effect of age was seen for both males and females which could be set equal. No influence of the shared environment could be detected. The specific genetic influences on the first and second measure of all haemostatic factors could be dropped in the best fitting model (see also Fig. 1). Furthermore, for tPA antigen and $\mathrm{vWF}$ the environmental component specific to the first measurement could be dropped from the model, indicating the emergence of some additional unique environmental influence at the second measurement. Two separate specific unique environmental factors were necessary for the two fibrinogen measurements suggesting different environmental influences (such as measurement error) acting on the variance at the two time points. For simplicity, Table 5 presents only the genetic and environmental influences for the baseline measurement (before stress) that was available in the largest number of subjects.

\section{Discussion}

In this study of 213 middle-aged male and female twin pairs we investigated potential sex differences in means and genetic architecture of fibrinogen, tPA antigen and vWF. Fibrinogen, an inflammatory marker, is one of the most consistent risk factors for CHD (6-8), and in this study slightly higher mean levels were found in women compared to men, as found earlier $(13,16)$. In concordance with intervention studies (27), we observed slightly higher fibrinogen levels in women on OCP compared to premenopausal women not on OCP. In addition, epidemiological studies (13) have also observed increased levels of fibrinogen in postmenopausal women, as we have in this study. After correcting for the effect of OCP and menopause, no difference in mean levels between males and females was observed, nor could any effect of age be seen. In contrast, tPA antigen, also a consistent risk factor for CHD, (9-12) showed lower mean levels in women as was also seen in an earlier study (16). In agreement with previous studies (27), we found that women on OCP had significantly lower levels of tPA antigen compared to premenopausal women not on OCP. Postmenopausal women showed increased levels of tPA antigen in this study, which is in concordance with a previous study (28). Although OCP and menopause influenced levels of tPA significantly, they could not explain the difference between males and females. In preliminary analysis, mean levels of tPA seemed to show a more rapid increase with age in females compared to males, however, after correcting for OCP and menopause, the effect of age was the same for males and females indicating no interaction between sex and age. Prospective studies have also identified vWF as a risk factor for CHD in the healthy population $(8,9)$. A large general population study reported higher levels of vWF in women compared to men (15), but in this study only a non significant trend was observed. The effect of age was significant with increasing levels of vWF in older individuals.

It is unclear to what extent these differences in mean levels of haemostatic risk factors relate to sex differences in ultimate risk for cardiovascular disease, but knowledge of the genetic architecture of these risk factors and their potential sex differences might help unravel the different pathways of the development of cardiovascular disease in males and females.

Genetic influences on fibrinogen, tPA antigen and vWF explained 39,67 and $72 \%$ of the variation, respectively. These figures are in line with earlier studies on the genetic influences on haemostatic factors (17-19) and potentially explain part of the genetic influence on $\operatorname{CHD}(4,5)$. Two of these studies are family studies $(17,19)$ and reported respective heritabilities of 34 and $35 \%$ for fibrinogen and are very close to our estimate of $39 \%$. A UK twin study reported a heritability of $44 \%$ for fibrinogen (18). In general, twin studies report higher heritabilities than family studies. For example, for tPA antigen, both family studies report an estimate of $27 \%$, which is lower than our estimate of $67 \%$, however, our estimate is very similar to the heritability estimate of $62 \%$ from the UK twin study (18). Our heritability of $72 \%$ for vWF is also very similar to the $75 \%$ estimated by the UK study (18), but higher than the $32 \%$ in one of the family studies (17). Part of this genetic effect could potentially be accounted for by the $\mathrm{ABO}$ blood group polymorphism, considering blood group explained up to $30 \%$ of the genetic variation in a small twin study (29). Since we are mainly interested in covariates that could potentially explain sex differences in variance components estimates, we adjusted for covariates such as OCP and menopausal status, assuming that effects of blood group would be fairly similar in males and females.

The power to detect a difference in magnitude of genetic estimates (i.e., heritability) between men and women depends on the distribution of MZ, DZ, male and female, and opposite sex twin pairs in the sample. Our sample of 210 twin pairs has $80 \%$ power $(\alpha=0.05)$ to detect a genetic difference in heritability of $20 \%$, if the lowest heritability is $45 \%$. If the lowest heritability would be $60 \%$, this same sample composition would have $80 \%$ power $(\alpha=0.05)$ to detect a difference of $15 \%$ and larger. This seems quite adequate for tPA and vWF in view of the heritabilities found, but power may have been somewhat low for fibrinogen. No difference in the magnitude of genetic or environmental effects or differences in genes between males and females on levels of fibrinogen, tPA or VWF could be detected. The advantage of model fitting using twin data is that the estimated heritability summarises all genes involved, both structural and regulatory genes, and is a good test for overall genetic sex differences. Then again, while twin studies are the ideal study design to measure these relatively important genetic and environmental factors to the variance of traits and diseases, one has to consider that, without actual measurement of specific genes or environments, it cannot attribute the sex difference in mean values to either of these factors (30).

A limitation of this study is the relative small sample size of the opposite sex twin pair group, which will have made it difficult to detect small differences in genetic and/or environmental effects. The lower correlation of the opposite sex twins compared to those of the same-sex dizygotic twins for fibrinogen 
( 0.12 vs. 0.21 males and 0.15 females) and vWF ( 0.26 vs. 0.40 males and 0.29 females) does suggest qualitative genetic differences, but not for tPA antigen (OS corr 0.32 vs. 0.30 males and 0.30 females). A study including a larger opposite sex twin group would have a better chance to detect this interesting indication of different genes operating in males compared to females.

To our knowledge this is the first study to investigate differences in genetic architecture between males and females for hae- mostatic risk factors of CHD. Although no quantitative or qualitative differences in genetic influence on fibrinogen, tPA antigen and $\mathrm{vWF}$ were observed, a trend of a lower genetic correlation between opposite sex twins compared to same-sex DZ twins could suggest a lack of power to detect subtle differences in gene expression between males and females. Unravelling of these sex differences in larger samples may help understand the pathophysiological mechanisms leading to (cardio)vascular disease.

\section{References}

1. Zdravkovic S, Wienke A, Pedersen NL, et al. Heritability of death from coronary heart disease: a 36-year follow-up of 20966 Swedish twins. J Intern Med 2002; 252: $247-54$.

2. Marenberg ME, Risch N, Berkman LF, et al. Genetic susceptibility to death from coronary heart disease in a study of twins. N Engl J Med 1994; 330: 1041-6. 3. Zdravkovic S, Wienke A, Pedersen NL, et al. Genetic influences on CHD-death and the impact of known risk factors: comparison of two frailty models. Behav Genet 2004; 34: 585-92.

4. Sing CF, Moll PP. Genetics of atherosclerosis. Annu Rev Genet 1990; 24: 171-87.

5. Schork NJ. Genetically complex cardiovascular traits. Origins, problems, and potential solutions. Hypertension 1997; 29: 145-9.

6. Meade TW, Mellows S, Brozovic M, et al. Haemostatic function and ischaemic heart disease: principal results of the Northwick Park Heart Study. Lancet 1986; 2: 533-7.

7. Kannel WB, Wolf PA, Castelli WP, et al. Fibrinogen and risk of cardiovascular disease. The Framingham Study. JAMA 1987; 258: 1183-6.

8. Folsom AR, Wu KK, Rosamond WD, et al. Prospective study of hemostatic factors and incidence of coronary heart disease: the Atherosclerosis Risk in Communities (ARIC) Study. Circulation 1997; 96: 1102-8.

9. Thögersen AM, Jansson JH, Boman K, et al. High plasminogen activator inhibitor and tissue plasminogen activator levels in plasma precede a first acute myocardial infarction in both men and women: evidence for the fibrinolytic system as an independent primary risk factor. Circulation 1998; 98: 2241-7.

10. Folsom AR, Aleksic N, Park E, et al. Prospective study of fibrinolytic factors and incident coronary heart disease: the Atherosclerosis Risk in Communities (ARIC) Study. Arterioscler Thromb Vasc Biol 2001; 21: 611-7.
11. Ridker PM, Vaughan DE, Stampfer MJ, et al. Endogenous tissue-type plasminogen activator and risk of myocardial infarction. Lancet 1993; 341: 1165-8. 12. Lowe GDO, Yarnell JWG, Sweetnam PM, et al. Fibrin D-dimer, tissue plasminogen activator, plasminogen activator inhibitor, and the risk of major ischaemic heart disease in the Caerphilly Study. Thromb Haemost 1998; 79: 129-33.

13. Lowe GD, Rumley A, Woodward M, et al. Epidemiology of coagulation factors, inhibitors and activation markers: the Third Glasgow MONICA Survey. I. Illustrative reference ranges by age, sex and hormone use. Br J Haematol 1997; 97: 775-84.

14. Kalaria VG, Zareba W, Moss AJ, et al. Gender-related differences in thrombogenic factors predicting recurrent cardiac events in patients after acute myocardial infarction. The THROMBO Investigators. Am J Cardiol 2000; 85: 1401-8.

15. Conlan MG, Folsom AR, Finch A, et al. Associations of factor VIII and von Willebrand factor with age, race, sex, and risk factors for atherosclerosis. The Atherosclerosis Risk in Communities (ARIC) Study. Thromb Haemost 1993; 70: 380-5.

16. Mennen LI, Balkau B, Charles MA, et al. Gender differences in the relation between fibrinogen, tissuetype plasminogen activator antigen and markers of insulin resistance: effects of smoking. D.E.S.I.R. Study Group. Data from an Epidemiological Study on Insulin Resistance Syndrome. Thromb Haemost 1999; 82: 1106-11.

17. Souto JC, Almasy L, Borrell M, et al. Genetic determinants of hemostasis phenotypes in Spanish families. Circulation 2000; 101: 1546-51.

18. de Lange M, Snieder H, Ariëns RA, et al. The genetics of haemostasis: a twin study. Lancet 2001; 357: $101-5$.

19. Freeman MS, Mansfield MW, Barrett JH, et al. Genetic contribution to circulating levels of hemostatic factors in healthy families with effects of known gen- etic polymorphisms on heritability. Arterioscler Thromb Vasc Biol 2002; 22: 506-10.

20. Snieder H, Boomsma DI, van Doornen LJP, et al. Heritability of respiratory sinus arrhythmia: dependency on task and respiration. Psychophysiology 1997; 34: $317-28$.

21. Snieder H, Huizink AC, Boomsma DI, et al. Influence of mental stress on fibrinogen, von Willebrand factor and tissue-type plasminogen activator antigen. Fibrinolysis 1996; 10 (Suppl 2): 137-9.

22. Hoegee-de Nobel E, Voskuilen M, Briet E, et al. A monoclonal antibody-based quantitative enzyme immunoassay for the determination of plasma fibrinogen concentrations. Thromb Haemost 1988; 60: 415-8.

23. Bos R, Hoegee-de Nobel E, Laterveer R, et al. A one-step enzyme immunoassay for the determination of total tissue-type plasminogen activator (t-PA) antigen in plasma. Blood Coagul Fibrinolysis 1992; 3: 303-7.

24. StataCorp Stata Statistical Software, release 7.0. 2001.

25. Boomsma D, Busjahn A, Peltonen L. Classical twin studies and beyond. Nat Rev Genet 2002; 3: 872-82.

26. Neale MC, Boker SM, Xie G, et al. Mx: statistical modeling. 1999; Fifth Edition.

27. Wiegratz I, Lee JH, Kutschera E, et al. Effect of four oral contraceptives on hemostatic parameters. Contraception 2004; 70: 97-106.

28. Gebara OC, Mittleman MA, Sutherland P, et al. Association between increased estrogen status and increased fibrinolytic potential in the Framingham Offspring Study. Circulation 1995; 91: 1952-8.

29. Orstavik KH, Magnus P, Reisner H, et al. Factor VIII and factor IX in a twin population. Evidence for a major effect of ABO locus on factor VIII level. Am J Hum Gen 1985; 37: 89-101.

30. Snieder H, Harshfield GA, Treiber FA. Heritability of blood pressure and hemodynamics in African- and European-American youth. Hypertension 2003; 41: 1196-201. 